\title{
EXISTENCE THEOREMS FOR STRONGLY COUPLED SYSTEMS OF PARTIAL DIFFERENTIAL EQUATIONS OVER BERNSTEIN CLASSES
}

\author{
BY RAY REDHEFFER ${ }^{1}$ AND WOLFGANG WALTER
}

Communicated by F. Brauer, June 23, 1976

If $f$ is an entire function of type $\sigma$, bounded on the real axis, a well-known theorem of Bernstein gives $\sup _{x \in \mathbf{R}}\left|f^{\prime}(x)\right| \leqslant \sigma \sup _{x \in \mathbf{R}}|f(x)|$. Our objective is to construct a Banach space, based on this theorem, in which the existence problem for broad classes of partial differential equations can be solved with surprising ease. The idea of using Bernstein's theorem in the study of coupled systems is due to Nickel [3].

1. Notation. We denote real or complex $m$-dimensional space by $\mathbf{R}^{m}$ or $\mathbf{C}^{m}$ respectively, and the norm of a vector $z$ in one of these spaces by $|z|$. The norm of any function $F: \mathbf{F} \rightarrow \mathbf{C}^{p}$ is $\|F\|=\sup _{\xi \in \mathbf{F}}|F(\xi)|$. We choose $\sigma \in \mathbf{R}^{m}$ fixed, $\sigma_{i} \geqslant 0$, and use $j$ as a multi-index; thus:

$$
D^{j}=\frac{\partial^{|j|}}{\partial x_{1}^{j_{1}} \partial x_{2}^{j_{2}} \cdots \partial x_{m}^{j_{m}}}, \quad \sigma^{j}=\sigma_{1}^{j_{1}} \sigma_{2}^{j_{2}} \cdots \sigma_{m}^{j_{m}} .
$$

2. The class $B(m, p, \sigma)$. The class $B(m, 1, \sigma)$ is the class of functions $f$ : $\mathbf{R}^{m} \rightarrow \mathbf{C}$ satisfying one of the following equivalent conditions:

(a) $f$ can be extended to a holomorphic function $f^{*}: \mathbf{C}^{m} \rightarrow \mathbf{C}$ so that, for all $z=x+i y \in \mathbf{C}^{m}$ and for some constant $M=M(f)$,

$$
\left|f^{*}(z)\right| \leqslant M \exp \left(\sigma_{1}\left|y_{1}\right|+\sigma_{2}\left|y_{2}\right|+\cdots+\sigma_{m}\left|y_{m}\right|\right) .
$$

(b) $f$ can be extended to a holomorphic function $f^{*}: \mathbf{C}^{m} \rightarrow \mathbf{C}$ so that, for all $z=x+i y \in \mathbf{C}^{m}$ and all multi-indices $j$,

$$
\left|D^{j} f^{*}(z)\right| \leqslant\|f\| \sigma^{j} \exp \left(\sigma_{1}\left|y_{1}\right|+\sigma_{2}\left|y_{2}\right|+\cdots+\sigma_{m}\left|y_{m}\right|\right)<\infty .
$$

(c) $f \in \mathbf{C}^{\infty}$ and $\left\|D^{j} f\right\| \leqslant\|f\| \sigma^{j}<\infty$ for all multi-indices $j$. That (b) $\Rightarrow$ (c) $\wedge$ (a) is obvious, and (c) $\Rightarrow$ (b) by Taylor's formula. Also, (a) $\Rightarrow$ (b) follows for $m=1$ from Bernstein's theorem and then in general by induction on $m$.

By $(\mathrm{a}), B(m, 1, \sigma)$ is a linear space which contains all functions

AMS (MOS) subject classifications (1970). Primary 35 A05, 35G05, 35J55, 35 K45, $35 \mathrm{~K} 55,35 \mathrm{R} 25$.

${ }^{1}$ Under auspices of the U.S. Special Program, Alexander von Humboldt Stiftung. 


$$
f(z)=f_{1}\left(z_{1}\right) f_{2}\left(z_{2}\right) \cdots f_{m}\left(z_{m}\right), \quad f_{i} \in B\left(1,1, \sigma_{i}\right),
$$

and which is closed under translation $z \rightarrow z-z_{0}$. The class $\bigcup_{\sigma} B(m, 1, \sigma)$ is closed under addition, multiplication, and substitutions $z \rightarrow A z-z_{0}$ where $z_{0}$ and the $m$ by $m$ matrix $A$ are constant.

A function $f=\left(f_{1}, f_{2}, \ldots, f_{p}\right)^{\text {tr }}$ from $\mathbf{R}^{m}$ to $\mathbf{C}^{p}$ is in $B(m, p, \sigma)$ if $f_{i} \in$ $B(m, 1, \sigma)$ for $i=1,2, \ldots, p$. Then (a) shows that $B(m, p, \sigma)$ is a linear space and (c) shows that $D^{j}$ is a bounded operator on $B(m, p, \sigma)$. By (c) or (a) the space is complete.

3. The Banach space $B(m, p, \sigma, \mathbf{D})$. Let $\mathbf{D} \subset \mathbf{R}^{n}$. The function $f: \mathbf{R}^{m} \times$ $\mathbf{D} \rightarrow \mathbf{C}^{p}$ belongs to $B(m, p, \sigma, \mathbf{D})$ if $f$ is continuous and bounded, and if $f(x, y)$ $\in B(m, p, \sigma)$ as a function of $x$ for each $y \in \mathrm{D}$. This is a Banach space with norm $\|f\|$ as in $\S 1$.

If $f \in B(m, p, \sigma, \mathrm{D})$, (c) indicates that all derivatives of $f(x, y)$ with respect to $x$ are bounded. Hence $D^{i} f(x, y)$ is a uniform limit of difference quotients, each of which is continuous; for instance, if $m=n=p=j=1$,

$$
\left|h^{-1}[f(x+h, y)-f(x, y)]-f_{x}(x, y)\right| \leqslant|h|\left|f_{x x}(x+\theta h, y)\right| \quad(h \neq 0) .
$$

This shows that $D^{i} f(x, y)$ is continuous. Referring to (c) again, we get the following

ThEOREM. $B(m, p, \sigma, \mathrm{D})$ is a Banach space and $D^{j}$ is a bounded operator on it.

4. A boundary-value problem. We write $z \in \mathbf{C}^{p}$ as a column $\left(z_{1}, z_{2}, \ldots, z_{p}\right)^{\mathrm{tr}}$. If $A$ is a $p$ by $p$ matrix it is assumed that the matrix norm is compatible with the norm in $\mathbf{C}^{p}$, in the sense $|A z| \leqslant|A||z|$. We also require $|z| \leqslant|\widetilde{z}|$ whenever $\left|z_{i}\right| \leqslant\left|\widetilde{z}_{i}\right|$.

Let $A_{j}(t)$ be $p$ by $p$ matrix-valued functions, continuous for $0 \leqslant t \leqslant T$, let $B=B(m, p, \sigma,[0, T])$, and let

$$
L u(x, t)=\sum_{|j| \leqslant k} A_{j}(t) D^{j} u(x, t)
$$

with $D^{j}$ as in $\S 1$. Then the following holds:

COROLlary 1. If $f \in B$ and $h \in B$, the boundary-value problem

$$
u_{t}=L u+h(x, t) \text { in } \mathbf{R}^{m} \times[0, T], \quad u(x, 0)=f(x, 0)
$$

has one and only one solution $u \in B$.

For proof, write the differential equation as $u=H+M u$ where

$$
H(x, t)=f(\dot{x}, 0)+\int_{0}^{t} h(x, \tau) d \tau, \quad M u(x, t)=\int_{0}^{t}(L u)(x, \tau) d \tau .
$$


Then $H \in B$, as is easily checked. Also, for $u \in B$,

$$
|L u(x, t)| \leqslant \sum_{|j| \leqslant k}\left|A_{j}(t)\right|\left\|D^{j} u\right\| \leqslant \sum_{|j| \leqslant k}\left|A_{j}(t)\right| \sigma^{j}\|u\|
$$

and, hence, $\|L u\| \leqslant K\|u\|$ for a constant $K$. Thus $\|M u\| \leqslant K T\|u\|$, and the conclusion follows from the contraction principle if $K T<1$. For $K T>1$, repeat the process.

5. Parabolic equations. Let $\mathbf{D} \subset \mathbf{R}^{n}$ be open, not necessarily bounded, and let $\Omega_{0}=\mathbf{D} \times(0, T], \Omega=\mathbf{R}^{m} \times \mathbf{D} \times(0, T]$. The parabolic boundaries are $\Gamma_{0}=\bar{\Omega}_{0}-\Omega_{0}$ and $\Gamma=\bar{\Omega}-\Omega$. For $u=\left(u_{1}, u_{2}, \ldots, u_{p}\right)^{\operatorname{tr}}$ as in $\S 4$ we define $L_{x}$ by (1) and also

$$
\begin{aligned}
\Delta_{y} u_{j} & =\sum_{i=1}^{n}\left(\frac{\partial}{\partial y_{i}}\right)^{2} u_{j}, \\
\Delta_{y} u & =\left(\Delta_{y} u_{1}, \Delta_{y} u_{2}, \ldots, \Delta_{y} u_{p}\right)^{\mathrm{tr}} .
\end{aligned}
$$

Thus, $L_{x}$ involves differentiation only with respect to $x$ and $\Delta_{y}$ only with respect to $y$. We set $B_{1}=B(m, p, \sigma, \overline{\mathbf{D}} \times[0, T])$.

CoRollary 2. Suppose $h \in B_{1}$, and suppose Green's function for the problem

$$
\Phi_{t}(y, t)=\Delta_{y} \Phi+g(y, t) \quad \text { in } \Omega_{0}, \quad \Phi=0 \text { on } \Gamma_{0}
$$

exists. Then the problem

$$
u_{t}=\Delta_{y} u+L_{x} u+h(x, y, t) \quad \text { in } \Omega, \quad u=0 \quad \text { on } \Gamma
$$

has one and only one solution $u \in B_{1}$.

For proof, let $G$ be the Green function and write (2) as

$$
u(x, y, t)=\int_{0}^{t} \int_{\mathrm{D}} G(y-\eta, t-\tau)\left[h(x, \eta, \tau)+\left(L_{x} u\right)(x, \eta, \tau)\right] d \eta d \tau .
$$

In an obvious notation, $u=H+M u$. From $|L u| \leqslant K\|u\|$ follows

$$
\|M u\| \leqslant K\|u\| \int_{0}^{t} \int_{\mathrm{D}} G(y-\eta, t-\tau) d \eta d \tau \leqslant K T\|u\|,
$$

and this gives the conclusion as in $\S 4$ above.

Clearly, these results can be generalized. In Corollary 2 , for example, the Laplacian can be replaced by an arbitrary elliptic operator for which Green's function exists, and inhomogeneous boundary conditions can be allowed. For alternative approaches to the theory of coupled systems, compare [1], [2] . 


\section{REFERENCES}

1. Lars Hörmander, Linear partial differential operators, Academic Press, New York; Springer-Verlag, Berlin, 1963. MR $28 \# 4221$.

2. S. G. Kreīn, Linear differential equations in Banach space, "Nauka", Moscow, 1967; English transl., Transl. Math. Monographs, vol. 29, Amer. Math. Soc., Providence, R.I., 1971. MR 40 \#508; 49 \#7548.

3. Karl Nickel, New results on strongly coupled systems of parabolic differential equations, Proc. Conf. (Univ. of Dundee, March, 1976), Dundee, Scotland (to appear).

MATHEMATISCHES INSTITUT I, UNIVERSITÄT KARLSRUHE (TH), POSTFACH 6380 D-7500 KARLSRUHE, FEDERAL REPUBLIC OF GERMANY 